\title{
Algebraic properties of Zappa-Szép products of semigroups and monoids
}

\author{
Rida-e Zenab ${ }^{1}$
}

Received: 14 May 2016 / Accepted: 5 May 2017 / Published online: 23 May 2017

(C) The Author(s) 2017. This article is an open access publication

\begin{abstract}
Direct, semidirect and Zappa-Szép products provide tools to decompose algebraic structures, with each being a natural generalisation of its predecessor. In this paper we examine Zappa-Szép products of monoids and semigroups and investigate generalised Greens relations $\mathcal{R}^{*}, \mathcal{L}^{*}, \widetilde{\mathcal{R}}_{E}$ and $\widetilde{\mathcal{L}}_{E}$ for these Zappa-Szép products. We consider a left restriction semigroup $S$ with semilattice of projections $E$ and define left and right actions of $S$ on $E$ and $E$ on $S$, respectively, to form the Zappa-Szép product $E \bowtie S$. We further investigate properties of $E \bowtie S$ and show that $S$ is a retract of $E \bowtie S$. We also find a subset $T$ of $E \bowtie S$ which is left restriction.
\end{abstract}

Keywords Left restriction semigroups · Generalised Green's relations - Semidirect products · Zappa-Szép products

\section{Introduction}

Semidirect products and their generalisation Zappa-Szép products have become well known tools to decompose algebraic structures such as semigroups over recent years. The aim of this paper is to study algebraic properties of Zappa-Szép products of semigroups. The Zappa-Szép products of semigroups were properly developed by Kunze [14] who gave applications of Zappa-Szép products to translational hulls, Bruck-Reilly extensions and Rees matrix semigroups. The Zappa-Szép products of semigroups involve actions of two semigroups on each other. These actions are closely

Communicated by Jean-Eric Pin.

Rida-e Zenab

ridaezenab@iba-suk.edu.pk

1 Department of Mathematics, Sukkur Institute of Business Administration, Sukkur, Pakistan 
related to the actions of Mealy machines (automata with output). For convenience of the reader we begin by recalling the basic definition of Zappa-Szép products of semigroups.

Definition 1.1 Let $S$ and $T$ be semigroups and suppose that we have maps

$$
T \times S \rightarrow S,(t, s) \mapsto t \cdot s \text { and } T \times S \rightarrow T,(t, s) \mapsto t^{s}
$$

such that for all $s, s^{\prime} \in S, t, t^{\prime} \in T$ :

$$
\begin{array}{ll}
(\mathrm{ZS} 1) t t^{\prime} \cdot s=t \cdot\left(t^{\prime} \cdot s\right) ; & (\mathrm{ZS} 3)\left(t^{s}\right)^{s^{\prime}}=t^{s s^{\prime}} \\
(\mathrm{ZS} 2) t \cdot\left(s s^{\prime}\right)=(t \cdot s)\left(t^{s} \cdot s^{\prime}\right) ; & (\mathrm{ZS} 4)\left(t t^{\prime}\right)^{s}=t^{t^{\prime} \cdot s} t^{\prime s}
\end{array}
$$

From (ZS1) and (ZS3), we see that $T$ acts on $S$ from the left and $S$ acts on $T$ from the right, respectively. Define a binary operation on $S \times T$ by

$$
(s, t)\left(s^{\prime}, t^{\prime}\right)=\left(s\left(t \cdot s^{\prime}\right), t^{s^{\prime}} t^{\prime}\right) .
$$

It is easy to see that this binary operation is associative. Thus $S \times T$ becomes a semigroup known as the (external) Zappa-Szép product of $S$ and $T$ and denoted by $S \bowtie T$.

Note that if one of the above actions is trivial (that is, one semigroup acts by the identity map), then the second action is by morphisms, and we obtain the semidirect product $S \rtimes T$ (if $S$ acts trivially) or $S \ltimes T$ (if $T$ acts trivially).

If $S$ and $T$ are monoids then the following four axioms are also required to hold:

$$
\begin{array}{ll}
\text { (ZS5) } t \cdot 1_{S}=1_{S} ; & (\mathrm{ZS} 7) 1_{T} \cdot s=s \\
\text { (ZS6) } t^{1}=t ; & (\mathrm{ZS} 8) 1_{T}^{s}=1_{T}
\end{array}
$$

From (ZS6) and (ZS7) we see that the actions are monoid actions and (ZS5) and (ZS8) are saying that the identities are fixed under the actions. Then $S \bowtie T$ becomes a monoid with identity $\left(1_{S}, 1_{T}\right)$.

If we replace monoids by groups in the above definition, then $S \bowtie T$ is a group (see $[21,25])$. It is well known that there is an equivalence between internal and external Zappa-Szép products of groups. A similar correspondence exists for monoids [14], as we now explain. If $Z$ is a monoid with submonoids $S$ and $T$ such that $Z=S T$ and every element $z \in Z$ has a unique decomposition as $z=s t$, where $s \in S$ and $t \in T$, then we say that $Z$ is the internal Zappa-Szép product of $S$ and $T$. If $Z=S \bowtie T$ is the external Zappa-Szép product of monoids $S$ and $T$, then putting

$$
S^{\prime}=\left\{\left(s, 1_{T}\right): s \in S\right\} \text { and } T^{\prime}=\left\{\left(1_{S}, t\right): t \in T\right\}
$$

we have that $S^{\prime}$ and $T^{\prime}$ are submonoids of $Z$, isomorphic to $S$ and $T$ respectively, such that $Z$ is the internal Zappa-Szép product of $S^{\prime}$ and $T^{\prime}$. Conversely if $Z=$ $S T$ is the internal Zappa-Szép product of submonoids $S$ and $T$ then uniqueness of 
decompositions and associativity enable us to show that $Z$ is isomorphic to an external Zappa-Szép product $S \bowtie T$. For semigroups there is no such universal correspondence between internal and external Zappa-Szép products and indeed not even for semidirect products, as remarked by Preston [20]. Preston went on to provide, by judicious adding of identities, a way to move between internal and external semidirect products of semigroups, but the correspondence is weaker than that in the case for monoids [20]. A similar correspondence in the case of Zappa-Szép products of semigroups is given in the thesis of the author [26].

In Sect. 2 we record the behaviour of the generalised Green's relations on the Zappa-Szép products of semigroups and monoids. Green's relations $\mathcal{R}, \mathcal{L}, \mathcal{H}, \mathcal{D}$ and $\mathcal{J}$ are well known equivalence relations that characterise the elements of a semigroup in terms of one and two-sided principal ideals they generate. The reader is referred to [12] for further details concerning Green's relations. We explain how generalised Green's relations may be used to characterise elements of non-regular semigroups.

The relation $\mathcal{R}^{*}\left(\mathcal{L}^{*}\right)$ on a semigroup $S$ is defined by the rule that for any $s, t \in S$, $s \mathcal{R}^{*} t\left(s \mathcal{L}^{*} t\right)$ if and only if for all $u, v \in S^{1}$ (the extension of $S$ by the addition of an identity element)

$$
u s=v s \text { if and only if } u t=v t(s u=s v \text { if and only if } t u=t v) .
$$

We note that $\mathcal{R}^{*}$ and $\mathcal{L}^{*}$ are equivalence relations. Also it is clear that $\mathcal{R}^{*}$ is a left congruence and $\mathcal{L}^{*}$ is a right congruence. We observe that an idempotent $e$ of $S$ acts as a left identity for its $\mathcal{R}^{*}$-class and a right identity for its $\mathcal{L}^{*}$-class. Also note that $\mathcal{R} \subseteq \mathcal{R}^{*}$ and $\mathcal{L} \subseteq \mathcal{L}^{*}$. If $S$ is regular then $\mathcal{R}=\mathcal{R}^{*}$ and $\mathcal{L}=\mathcal{L}^{*}$. A useful observation is that unlike Green's relations, the relations $\mathcal{R}^{*}$ and $\mathcal{L}^{*}$ need not commute.

Now we turn our attention to the relations that play an important role to define a left (right, two-sided) restriction semigroup. Let $S$ be a semigroup and let $E$ be a subset of $E(S)$, where $E(S)$ denotes the set of idempotents of $S$. The relation $\widetilde{\mathcal{R}}_{E}$ on $S$ is defined as for any $s, t \in S, s \widetilde{\mathcal{R}}_{E} t$ if and only if for all $e \in E$,

$$
e s=s \text { if and only if } e t=t,
$$

that is, $s$ and $t$ have same set of left identities in $E$. Dually, $s \widetilde{\mathcal{L}}_{E} t$ if and only $s$ and $t$ have same set of right identities in $E$.

Certainly $\widetilde{\mathcal{R}}_{E}$ and $\widetilde{\mathcal{L}}_{E}$ are equivalence relations, however, unlike the case for $\mathcal{R}, \mathcal{L}$, $\mathcal{R}^{*}$ and $\mathcal{L}^{*}$, it is not true that $\widetilde{\mathcal{R}}_{E}$ and $\widetilde{\mathcal{L}}_{E}$ are respectively left and right compatible.

We note that any $e \in E$ is a left (right) identity for its $\widetilde{\mathcal{R}}_{E}$-class ( $\left(\widetilde{\mathcal{L}}_{E}\right.$-class). This is because, if $s \in S$ and $s \widetilde{\mathcal{R}}_{E} e$, then since $e \in E$,

$$
e e=e \Rightarrow e s=s
$$

In fact we can say more about it as we see in the following lemma.

Lemma 1.2 [15] Let $S$ be a semigroup and $E \subseteq E(S)$. If $s \in S$ and $e \in E$, then $s \widetilde{\mathcal{R}}_{E} e\left(s \widetilde{\mathcal{L}}_{E}\right.$ e) if and only if es $=s(s e=s)$ and for all $f \in E$,

$$
f s=s \Rightarrow f e=e(s f=s \Rightarrow e f=e) .
$$


It is easy to see that $\mathcal{R} \subseteq \mathcal{R}^{*} \subseteq \widetilde{\mathcal{R}}_{E}$ and $\mathcal{L} \subseteq \mathcal{L}^{*} \subseteq \widetilde{\mathcal{L}}_{E}$. If $S$ is regular and $E=E(S)$, then the foregoing inclusions are replaced by equalities. More generally, if $e, f \in E$ then $e \widetilde{\mathcal{R}}_{E} f$ if and only if $e \mathcal{R} f$ and $e \widetilde{\mathcal{L}}_{E} f$ if and only if $e \mathcal{L} f$. In general, however, the inclusions are strict.

We denote idempotents in the $\widetilde{\mathcal{R}}_{E}$-class and $\widetilde{\mathcal{L}}_{E}$-class of an element $s$ by $s^{+}$and $s^{*}$, respectively. Note that $s^{+}$and $s^{*}$ are unique if $E$ is a semilattice. We note that for any distinguished idempotents $s^{+}$and $s^{*}$ in $\widetilde{\mathcal{R}}_{E}$-class and $\widetilde{\mathcal{L}}_{E}$-class of $s$ respectively, we have that

$$
s^{+} s=s \text { and } s s^{*}=s .
$$

In Sect. 3 we consider the Zappa-Szép product of a left restriction semigroup and a semilattice. Restriction semigroups and their one-sided versions have been developed from various points of view and under different names since the 1960s. Left restriction semigroups appeared in their own right for the first time in the work of Trokhimenko [24]. They were studied as type SL2 $\gamma$-semigroups in early 1980s by Batbedat and Fountain $[1,2]$. Lawson studied (left) restriction semigroups as the idempotent connected Ehresmann semigroups by drawing connection between semigroup theory and category theory [15]. In the last decade they were studied by Jackson and Stokes [13] in the guise of (left) twisted C-semigroups motivated by consideration of closure operators, and by Manes [16] as guarded semigroups which arose from the restriction categories of Cockett and Lack [4].

In recent years, an analogous theory to that of structure theory of inverse semigroups has been developed in the broader setting of restriction semigroups. One of the fundamental results in the structure theory of semigroups is that every inverse semigroup is an idempotent separating homomorphic image of a 'proper' inverse semigroup where a proper inverse semigroup is isomorphic to a so called ' $P$-semigroup' and embeds into a semidirect product of a semilattice by a group [17-19]. For (left) restriction semigroups, many authors have produced work using constructions similar (at least on the surface) to those of McAlister, replacing groups by monoids of various kinds. Covering results for restriction semigroups are known due to Cornock, Fountain, Gomes and Gould $[3,5,7,8]$. The structure of proper restriction semigroups is determined by analogues of $P$-semigroups. It has been shown that every restriction semigroup is embeddable into an almost left factorisable restriction semigroup. Also every restriction semigroup has a cover which is embeddable into a $W$-product (a special kind of semidirect product) [22,23]. Recent development in this theory reveals that restriction semigroups have become a topic of lively research interest and in providing the common frame work for answering different questions in algebra, they will continue to be a key topic.

A left restriction semigroup $S$ is a unary semigroup $\left(S, \cdot{ }^{+}\right)$, where $(S, \cdot)$ is a semigroup and ${ }^{+}$is a unary operation such that the following identities hold:

$$
s^{+} s=s, s^{+} t^{+}=t^{+} s^{+},\left(s^{+} t\right)^{+}=s^{+} t^{+} \text {and } s t^{+}=(s t)^{+} s .
$$


Putting $E=\left\{s^{+}: s \in S\right\}$, it is easy to see that $E$ is a semilattice (semigroup with commuting idempotents). We call $E$ the semilattice of projections (formerly distinguished semilattice) of $S$.

Dually right restriction semigroups are defined where in this case the unary operation is denoted by * A restriction semigroup is a bi-unary semigroup $S$ which is both left restriction and right restriction and which also satisfies the linking identities

$$
\left(s^{+}\right)^{*}=s^{+} \text {and }\left(s^{*}\right)^{+}=s^{*} \text {. }
$$

We remark that an inverse semigroup is left restriction, where we define $s^{+}=s s^{-1}$ (and also right restriction where $s^{*}=s^{-1} s$ ). Also monoids are left restriction and known as reduced left restriction monoids with trivial semilattice of projections.

A left restriction semigroup $S$ is equipped with a partial order $\leq$ defined by the rule that for any $s, t \in S, s \leq t$ if and only if $s=s^{+} t$ [11]. It is easy to check that $\leq$ is compatible on both sides in $S$. The following technical result will be useful in the subsequent sections of this paper.

Lemma 1.3 [9] If $S$ is left restriction semigroup with semilattice of projections $E$, then for any $s, t \in S$,

(i) $\left(s^{+}\right)^{+}=s^{+}$;

(ii) $(s t)^{+} \leq s^{+}$;

(iii) $\left(s t^{+}\right)^{+}=(s t)^{+}$.

We refer the reader to [11] for a detailed study on left (right, two-sided) restriction semigroups.

The following lemma provides an alternative characterisation for left restriction semigroups.

Lemma 1.4 [9] Let $\left(S, \cdot,^{+}\right)$be a unary semigroup. Then $S$ is left restriction with semilattice of projections $E$ if and only if

(i) $E$ is a semilattice;

(ii) every $\widetilde{\mathcal{R}}_{E}$-class contains an idempotent of $E$;

(iii) the relation $\widetilde{\mathcal{R}}_{E}$ is a left congruence and

(iv) the left ample condition holds, that is, for all $s \in S$ and $e \in E$,

$$
s e=(s e)^{+} s
$$

The dual result holds for right restriction semigroups and the relation $\widetilde{\mathcal{L}}_{E}$.

\section{Generalised Green's relations on Zappa-Szép products}

Green's relations $\mathcal{R}$ and $\mathcal{L}$ on a Zappa-Szép product of semigroups are characterised by Kunze [14]. We aim to characterise generalised Green's relations in this section. We consider Zappa-Szép products of semigroups and monoids and record the behaviour of the relations $\mathcal{R}^{*}, \mathcal{L}^{*}, \widetilde{\mathcal{R}}_{E}$ and $\widetilde{\mathcal{L}}_{E}$. The following is immediate from the definitions. 
Lemma 2.1 If $Z=S \bowtie T$ is a Zappa-Szép product of monoids $S$ and $T$, then $(a, b) \mathcal{R}^{*}(c, d)$ if and only if for all $x, u \in S$ and for all $y, v \in T$

$$
\begin{array}{ll}
x(y \cdot a)=u(v \cdot a) & \text { and } y^{a} b=v^{a} b \\
\Leftrightarrow x(y \cdot c)=u(v \cdot c) & \text { and } y^{c} d=v^{c} d .
\end{array}
$$

Proposition 2.2 Suppose $Z=S \bowtie T$ is a Zappa-Szép product of monoids $S$ and $T$. Then the following hold:

(1) if $b, d \in T$ and $b \mathcal{R}^{*} d$, then $(a, b) \mathcal{R}^{*}(a, d)$ in $Z$ for $a \in S$;

(2) if $a, c \in S$ and $a \mathcal{L}^{*} c$, then $(a, b) \mathcal{L}^{*}(c, b)$ in $Z$ for $b \in T$.

Proof (1) Suppose that $b \mathcal{R}^{*} d$ for some $b, d \in T$. Let $x, u \in S$ and $y, v \in T$, then for $a \in S$

$$
\begin{array}{lll}
x(y \cdot a)=u(v \cdot a) & \text { and } & y^{a} b=v^{a} b \\
\Leftrightarrow x(y \cdot a)=u(v \cdot a) & \text { and } & y^{a} d=v^{a} d \text { because } b \mathcal{R}^{*} d .
\end{array}
$$

By Lemma $2.1(a, b) \mathcal{R}^{*}(a, d)$ in $Z$.

The proof of (2) is dual.

Proposition 2.3 Let $S, T$ be monoids and $Z=S \bowtie T$ be the Zappa-Szép product of $S$ and T. Then

(1) $(a, b) \mathcal{R}^{*}(c, d)$ in $Z$ implies a $\mathcal{R}^{*} c$ in $S$;

(2) $(a, b) \mathcal{L}^{*}(c, d)$ in $Z$ implies $b \mathcal{L}^{*} d$ in $T$.

Proof (1) Suppose $(a, b) \mathcal{R}^{*}(c, d)$ in $Z$. To show that $a \mathcal{R}^{*} c$ in $S$, let $x, u \in S$ be such that

$$
x a=u a .
$$

Then

$$
x(1 \cdot a)=u(1 \cdot a) \text { and } 1^{a} b=1^{a} b,
$$

so by Lemma 2.1, $x(1 \cdot c)=u(1 \cdot c)$, that is, $x c=u c$. Thus together with the opposite direction, we obtain $a \mathcal{R}^{*} c$ in $S$.

The proof for $\mathcal{L}^{*}$ is dual.

Definition 2.4 Let $S$ and $T$ be semigroups and $\alpha: S \rightarrow T$ be a map from $S$ to $T$. We define $\operatorname{Ker} \alpha$ to be the relation

$$
\operatorname{Ker} \alpha=\{(s, t) \in S \times S: s \alpha=t \alpha\} .
$$

For more complete results, we now consider the reverse semidirect product $S \ltimes T$ of $S$ and $T$ and use Lemma 2.1 in the context when one of the actions is trivial, because in the Zappa-Szép product $S \bowtie T$ of monoids (semigroups) $S$ and $T$ if one of the actions 
is trivial (that is, one semigroup acts by the identity map), then the second action is by morphisms, and we obtain the semidirect product $S \rtimes T$ (if $S$ acts trivially) or $S \ltimes T$ (if $T$ acts trivially).

Proposition 2.5 Suppose $Z=S \ltimes T$ is a semidirect product of monoids $S$ and $T$, where $T$ is right cancellative. Also suppose that for any a, $c \in S$, a $\mathcal{R}^{*} c$ implies $\operatorname{Ker} a=\operatorname{Ker} c$ (where Ker $a$ is the kernel of the map induced by the right action of a). Then a $\mathcal{R}^{*} c$ in $S$ implies that $(a, b) \mathcal{R}^{*}(c, d)$ in $Z$ for all $b, d \in T$.

Proof Suppose $a \mathcal{R}^{*} c$ in $S$ and let $x, u \in S$ and $y, v \in T$. Then

$$
\begin{aligned}
& x a=u a \text { and } y^{a} b=v^{a} b \\
& \Leftrightarrow x a=u a \text { and } y^{a}=v^{a} \quad \text { because } \mathrm{T} \text { is cancellative } \\
& \Leftrightarrow x c=u c \text { and } y^{c}=v^{c} \quad \text { because } a \mathcal{R}^{*} c \text { and Ker } a=\operatorname{Ker} c \\
& \Leftrightarrow x c=u c \text { and } y^{c} d=v^{c} d \text { because } T \text { is cancellative. }
\end{aligned}
$$

Thus Lemma 2.1 gives $(a, b) \mathcal{R}^{*}(c, d)$ in $Z$.

Corollary 2.6 Suppose $Z=S \ltimes T$ is a semidirect product of monoids $S$ and $T$, where $T$ is right cancellative. Also suppose that $S$ acts on $T$ injectively. Then a $\mathcal{R}^{*} c$ in $S$ implies that $(a, b) \mathcal{R}^{*}(c, d)$ in $Z$ for all $b, d \in T$.

Definition 2.7 Let $M$ be a monoid and $X$ be a set. An action of $M$ on $X$ is called faithful if for $m, n \in M$ with $m \neq n$, there exists an $x \in X$ such that $m \cdot x \neq n \cdot x$.

Theorem 2.8 Let $Z=S \bowtie T$ be a Zappa-Szép product of monoids $S$ and $T$, where $T$ is right cancellative. Suppose $S$ acts faithfully on the right of $T$ and for any $a, c \in S$, $a \mathcal{R}^{*} c$ implies $\operatorname{Ker} a=\operatorname{Ker} c$. Then $a \mathcal{R}^{*} c$ in $S$ implies that $(a, b) \mathcal{R}^{*}(c, d)$ in $Z$ for all $b, d \in T$.

Proof Suppose $(a, b),(c, d) \in Z$ and $a \mathcal{R}^{*} c$ in $S$. To show that $(a, b) \mathcal{R}^{*}(c, d)$ in $Z$, let $x, u \in S$ and $y, v \in T$ be such that

$$
x(y \cdot a)=u(v \cdot a) \text { and } y^{a} b=v^{a} b .
$$

Then $y^{a}=v^{a}$, as $T$ is right cancellative. Also as $\operatorname{Ker} a=\operatorname{Ker} c$, we have $y^{c}=v^{c}$ and thus

$$
y^{c} d=v^{c} d
$$

Now for any $z \in T$,

$$
\left(z^{x} y\right)^{a}=z^{x(y \cdot a)} y^{a}=z^{u(v \cdot a)} v^{a}=\left(z^{u} v\right)^{a}
$$

and so as $\operatorname{Ker} a=\operatorname{Ker} c$,

$$
\left(z^{x} y\right)^{c}=\left(z^{u} v\right)^{c}
$$


gives

$$
z^{x(y \cdot c)} y^{c}=z^{u(v \cdot c)} v^{c}
$$

But $y^{c}=v^{c}$ and $T$ is right cancellative, therefore

$$
z^{x(y \cdot c)}=z^{u(v \cdot c)} .
$$

As this is true for any $z \in T$, and $S$ acts faithfully, we have

$$
x(y \cdot c)=u(v \cdot c) .
$$

Together with the opposite argument, Lemma 2.1 gives $(a, b) \mathcal{R}^{*}(c, d)$ in $Z$.

Theorem 2.9 Let $S, T$ be monoids and let $Z=S \bowtie T$ be a Zappa-Szép product of $S$ and $T$. Let $E \subseteq E(S)$ and $F \subseteq E(T)$. Put

$$
\bar{E}=\{(e, 1): e \in E\} \text { and } \bar{F}=\{(1, f): f \in F\} .
$$

Then $\bar{E}$ and $\bar{F}$ are sets of idempotents in $Z$ and

(1) $(a, b) \widetilde{\mathcal{R}}_{\bar{E}}(c, d)$ in $Z$ if and only if a $\widetilde{\mathcal{R}}_{E} c$ in $S$;

(2) $(a, b) \widetilde{\mathcal{L}}_{\bar{F}}(c, d)$ in $Z$ if and only if $b \widetilde{\mathcal{L}}_{F} d$ in $T$.

Proof It is easy to check that $\bar{E} \cup \bar{F} \subseteq E(Z)$.

(1) Suppose $(a, b) \widetilde{\mathcal{R}}_{\bar{E}}(c, d)$ in $Z$. Let $e \in E$. Then

$$
\begin{array}{rlr}
e a=a & \Leftrightarrow e(1 \cdot a) \quad=a & \text { by action of } T \text { on } S \\
& \Leftrightarrow(e, 1)(a, b)=(a, b) \\
& \Leftrightarrow(e, 1)(c, d)=(c, d) \quad \text { because }(a, b) \widetilde{\mathcal{R}}_{\bar{E}}(c, d) \\
& \Leftrightarrow e(1 \cdot c) \quad=c \\
& \Leftrightarrow e c & =c .
\end{array}
$$

Hence $a \widetilde{\mathcal{R}}_{E} c$ in $S$.

Conversely suppose that $a \widetilde{\mathcal{R}}_{E} c$ in $S$ and let $(a, b) \in Z$. Then for $(e, 1) \in \bar{E}$ we have

$$
\begin{aligned}
& (e, 1)(a, b)=(a, b) \\
& \Leftrightarrow\left(e(1 \cdot a), 1^{a} b\right)=(a, b) \\
& \Leftrightarrow(e a, b) \quad=(a, b) \\
& \Leftrightarrow e a \quad=a \\
& \Leftrightarrow e c \quad=c \quad \text { because } a \widetilde{\mathcal{R}}_{E} c \\
& \Leftrightarrow(e c, d) \quad=(c, d) \\
& \Leftrightarrow\left(e(1 \cdot c), 1^{c} d\right)=(c, d) \\
& \Leftrightarrow(e, 1)(c, d) \quad=(c, d) \text {. }
\end{aligned}
$$

Hence $(a, b) \widetilde{\mathcal{R}}_{\bar{E}}(c, d)$.

The proof for $\widetilde{\mathcal{L}}_{E}$ is dual. 
Theorem 2.10 Let $S$ and $T$ be semigroups and $Z=S \bowtie T$ be a Zappa-Szép product of $S$ and $T$. Let $E \subseteq E(S)$ and $F \subseteq E(T)$. Suppose there exists a right identity e for $S$ and a left identity $f$ for $T$ such that

$$
\begin{aligned}
& b \cdot e=e, b^{e}=b \text { for all } b \in T \\
& f \cdot a=a, f^{a}=f \text { for all } a \in S .
\end{aligned}
$$

Put

$$
\bar{E}=\{(h, f): h \in E\} \text { and } \bar{F}=\{(e, g): g \in F\} .
$$

Then $\bar{E}$ and $\bar{F}$ are sets of idempotents in $Z$ and

(i) $(a, b) \widetilde{\mathcal{L}}_{\bar{F}}(c, d) \Rightarrow b \widetilde{\mathcal{L}}_{F} d$ and $(a, b) \widetilde{\mathcal{R}}_{\bar{E}}(c, d) \Rightarrow a \widetilde{\mathcal{R}}_{E} c$;

(ii) $(a, b) \mathcal{L}^{*}(c, d) \Rightarrow b \mathcal{L}^{*} d$ and $(a, b) \mathcal{R}^{*}(c, d) \Rightarrow a \mathcal{R}^{*} c$.

Proof We first check that $\bar{E}$ and $\bar{F}$ are sets of idempotents in $Z$. For this let $(h, f) \in \bar{E}$. Then

$$
\begin{array}{rlrl}
(h, f)(h, f) & =\left(h(f \cdot h), f^{h} f\right) & \\
& =(h h, f f) \quad \text { using (RLI) } \\
& =(h, f) . &
\end{array}
$$

Thus $\bar{E} \subseteq E(Z)$ and similarly $\bar{F} \subseteq E(Z)$.

(i) Let $(a, b),(c, d) \in Z$ and suppose that $(a, b) \widetilde{\mathcal{L}}_{\bar{F}}(c, d)$. Let $g \in F$ be such that $b g=b$. Then $b^{e} g=b$ using (RLI). Now as $b \cdot e=e$ and $a e=a$, we have

$$
\begin{aligned}
& (a, b)(e, g)=(a, b) \\
\Rightarrow & (c, d)(e, g)=(c, d) \quad \text { because }(a, b) \widetilde{\mathcal{L}}_{\bar{E}}(c, d) \\
\Rightarrow & c(d \cdot e)=c \text { and } d^{e} g=d,
\end{aligned}
$$

giving $d g=d$. Together with the opposite direction, we obtain $b \widetilde{\mathcal{L}}_{F} d$.

Dually $(a, b) \widetilde{\mathcal{R}}_{\bar{E}}(c, d)$ implies $a \widetilde{\mathcal{R}}_{E} c$.

(ii) Suppose that $(a, b) \mathcal{R}^{*}(c, d)$ and let $u, v \in S^{1}$ be such that $u a=v a$. Then,

$$
\begin{aligned}
u(f \cdot a) & =v(f \cdot a) \quad \text { using }(\mathrm{RLI}) \\
\Rightarrow(u, f)(a, b) & =(v, f)(a, b) \\
\Rightarrow(u, f)(c, d) & =(v, f)(c, d) \text { because }(a, b) \mathcal{R}^{*}(c, d) \\
\Rightarrow u(f \cdot c) & =v(f \cdot c) \\
\Rightarrow u c \quad & =v c .
\end{aligned}
$$


Further,

$$
\begin{aligned}
a & =v a \\
& =v(f \cdot a) \\
\Rightarrow(a, b) & =(v, f)(a, b) \\
\Rightarrow(c, d) & =(v, f)(c, d) \text { because }(a, b) \mathcal{R}^{*}(c, d) \\
\Rightarrow c \quad & =v(f \cdot c) \\
\Rightarrow c \quad & =v c .
\end{aligned}
$$

Together with the dual, we obtain $a \mathcal{R}^{*} c$.

Dually $(a, b) \mathcal{L}^{*}(c, d)$ implies that $b \mathcal{L}^{*} d$.

\section{Zappa-Szép product of a left restriction semigroup and a semilattice}

In this section we consider a left restriction semigroup with semilattice of projections $E$. By defining a left action of $S$ on $E$ and a right action of $E$ on $S$, we see that $E \bowtie S$ becomes a Zappa-Szép product. This result was mentioned without proof in [10], as an example of a Zappa-Szép product. We include the full argument in this paper for the sake of completeness. Also we notice that $E \bowtie S$ is not itself left restriction but it contains a subsemigroup $T$ which is left restriction. Moreover, $S$ is a retract of $Z=E \bowtie S$.

Lemma 3.1 Let $S$ be a left restriction semigroup with semilattice of projections $E$. Define an action of $S$ on $E$ by $s \cdot e=(s e)^{+}$and an action of $E$ on $S$ by $s^{e}=s e$. Then $Z=E \bowtie S$ is a Zappa-Szép product of $S$ and $E$.

Proof We check that these two actions satisfy the axioms of a Zappa-Szép product.

(ZS1) For $s_{1}, s_{2} \in S$ and $e \in E$

$$
\begin{aligned}
s_{1} \cdot\left(s_{2} \cdot e\right) & =s_{1} \cdot\left(s_{2} e\right)^{+} \\
& =\left(s_{1}\left(s_{2} e\right)^{+}\right)^{+} \\
& =\left(s_{1}\left(s_{2} e\right)\right)^{+} \\
& =\left(\left(s_{1} s_{2}\right) e\right)^{+} \\
& =s_{1} s_{2} \cdot e .
\end{aligned}
$$

Hence (ZS1) holds.

(ZS2) Let $s \in S$ and $e, f \in E$. Then

$$
\begin{aligned}
(s \cdot e)\left(s^{e} \cdot f\right) & =(s e)^{+}(s e \cdot f) \\
& =(s e)^{+}((s e) f)^{+} \\
& =((s e) f)^{+} \quad \text { using Lemma } 1.3 \text { that }(a b)^{+} \leq a^{+} \text {for any } a, b \in S \\
& =(s(e f))^{+} \\
& =s \cdot e f .
\end{aligned}
$$

Thus (ZS2) holds. 
(ZS3) For $s \in S$ and $e, f \in E$, we have

$$
\left(s^{e}\right)^{f}=(s e)^{f}=(s e) f=s(e f)=s^{e f} .
$$

Hence (ZS3) holds.

(ZS4) For $s_{1}, s_{2} \in S, e \in E$

$$
\begin{aligned}
s_{1}^{s_{2} \cdot e} s_{2} e & =s_{1}^{\left(s_{2} e\right)^{+}}\left(s_{2} e\right) \\
& =s_{1}\left(s_{2} e\right)^{+} s_{2} e \\
& =s_{1}\left(s_{2} e\right) \\
& =\left(s_{1} s_{2}\right) e \\
& =\left(s_{1} s_{2}\right)^{e} .
\end{aligned}
$$

Hence (ZS4) holds. Thus $Z=E \bowtie S$ is a Zappa-Szép product under the binary operation

$$
(e, s)(f, t)=\left(e(s f)^{+}, s f t\right) .
$$

We remark that if $E$ acts on $S$ trivially, that is, $s^{e}=s$, then $S$ acts on $E$ by morphisms as

$$
s \cdot(e f)=(s e f)^{+}=\left((s e)^{+} s f\right)^{+}=(s e)^{+}(s f)^{+}=(s \cdot e)(s \cdot f)
$$

and so we obtain the semidirect product $E \rtimes S$.

If $S$ is left restriction, then the Zappa-Szép product $E \bowtie S$ obtained as above is the standard Zappa-Szép product of $E$ and $S$.

We now compute the set of idempotents of $Z=E \bowtie S$.

Lemma 3.2 Let $S$ be a left restriction semigroup with semilattice of projections $E$. Suppose that $Z=E \bowtie S$ is the standard Zappa-Szép product of $S$ and E. Then

$$
E(Z)=\left\{(e, s): e \leq s^{+}, s=s e s\right\} .
$$

Also $\bar{E}=\{(e, e): e \in E\}$ is a semilattice isomorphic to $E$ and if $E(S)=E$, then $\bar{E}=E(Z)$.

Proof Let $(e, s) \in Z$. Then

$$
\begin{aligned}
(e, s) \in E(Z) & \Leftrightarrow(e, s)^{2}=(e, s) \\
& \Leftrightarrow(e, s)(e, s)=(e, s) \\
& \Leftrightarrow\left(e(s e)^{+}, \text {ses }\right)=(e, s) \\
& \Leftrightarrow e=e(s e)^{+} \text {and } s=\text { ses. }
\end{aligned}
$$

Now $s=\operatorname{ses} \Rightarrow s \mathcal{R}$ se $\widetilde{\mathcal{R}}_{E}(s e)^{+}$, so that $s^{+}=(\text {se })^{+}$. Hence

$$
\begin{aligned}
(e, s) \in E(Z) & \Leftrightarrow\left(e s^{+}, \text {ses }\right)=(e, s) \\
& \Leftrightarrow e \leq s^{+} \quad \text { and } \operatorname{ses}=s .
\end{aligned}
$$


Also $\bar{E} \subseteq E(Z)$. It is easy to check that $\bar{E}$ is a semilattice isomorphic to $E$.

Now if $E(S)=E$, then $\bar{E}=E(Z)$, for if $(e, s) \in E(Z)$, then from $s=$ ses we obtain $s e=$ sese $=(s e)^{+}=s^{+}$. Also we have

$$
s=s e s=(s e) e s=s^{+} e s=e s
$$

giving $s^{+} \leq e$, so that as $e \leq s^{+}$it follows that $s^{+}=e$. Hence $s=$ ses $=s^{2}=s^{+}=e$.

We now record some of the properties of $Z=E \bowtie S$.

Proposition 3.3 Let $S$ be a left restriction semigroup with semilattice of projections $E$. Suppose that $Z=E \bowtie S$ is the standard Zappa-Szép product of $S$ and $E$. Let $(e, s) \in Z$, then:

(a) $\bar{E}=\{(e, e): e \in E\}$ is a semilattice isomorphic to $E(S)$;

(b) there is a semigroup epimorphism $\alpha: Z \rightarrow S$ separating the idempotents of $\bar{E}$;

(c) $(g, g)(e, s)=(e, s)$ if and only if $g e=e$ and $e s=s$;

(d) $(e, s)$ has a left identity in $\bar{E}$ if and only if es $=s$; in this case $(e, s) \widetilde{\mathcal{R}}_{\bar{E}}(e, e)$.

(e) $(e, s)(f, f)=(e, s)$ if and only if $e \leq s^{+}, s=s f$;

(f) for $(e, s) \in Z,(e, s) \widetilde{\mathcal{L}}_{\bar{E}}(f, f)$ where $(f, f) \in \bar{E}$ if and only if $e \leq s^{+}$and $s \widetilde{\mathcal{L}}_{E} f$

$(g)(g, g) \widetilde{\mathcal{R}}_{\bar{E}}(e, s) \widetilde{\mathcal{L}}_{\bar{E}}(f, f)$ for some $g, f \in E$ implies $(e, s)=\left(s^{+}, s\right)$.

Further, there is a canonical embedding of $S$ into $Z=E \bowtie S$ under $s \mapsto\left(s^{+}, s\right)$.

Proof (a) From Lemma 3.2, we know that $\bar{E}$ is a semilattice isomorphic to $E(S)$.

(b) Define $\alpha: Z \rightarrow S$ by $(e, s) \alpha=e s$. Clearly $\alpha$ is a surjection. To check that $\alpha$ is a homomorphism, let $(e, s),(f, t) \in Z$. Then

$$
\begin{aligned}
((e, s)(f, t)) \alpha & =\left(e(s f)^{+}, s f t\right) \alpha \\
& =e(s f)^{+} s f t \\
& =e(s f) t \\
& =e s f t \\
& =(e, s) \alpha(f, t) \alpha .
\end{aligned}
$$

Thus $\alpha$ is a homomorphism. Also for any $(e, e),(f, f) \in \bar{E}$, we have

$$
(e, e) \alpha=(f, f) \alpha \Leftrightarrow e=f
$$

and so $\alpha$ separates idempotents of $\bar{E}$.

(c) Let $(e, s) \in Z$ and $(g, g) \in \bar{E}$. Then

$$
\begin{aligned}
& (g, g)(e, s)=(e, s) \\
\Leftrightarrow & (g(g e), g e s)=(e, s) \\
\Leftrightarrow & g e=e \quad \text { and } g e s=s \\
\Leftrightarrow & g e=e \quad \text { and } e s=s .
\end{aligned}
$$


(d) Suppose now $(e, s) \widetilde{\mathcal{R}}_{\bar{E}}(h, h)$. By (c) we have $e s=s$.

Conversely, if $e s=s$, we note that $(e, e)$ is a left identity of $(e, s)$ as

$$
(e, e)(e, s)=(e, e s)=(e, s) .
$$

Next suppose that $(g, g) \in \bar{E}$ exists with $(g, g)(e, s)=(e, s)$. Then $g e=e$ from (c), so that, as $E \cong \bar{E}$, we have $(g, g)(e, e)=(e, e)$. Hence $(e, e) \widetilde{\mathcal{R}}_{\bar{E}}(e, s)$.

(e) For $(e, s) \in Z$ and $(f, f) \in \bar{E}$,

$$
\begin{aligned}
(e, s)(f, f)=(e, s) & \Leftrightarrow\left(e(s f)^{+}, s f f\right)=(e, s) \\
& \Leftrightarrow e(s f)^{+}=e \quad \text { and } s f=s \\
& \Leftrightarrow e \leq s^{+} \quad \text { and } s f=s .
\end{aligned}
$$

(f) Let $(e, s) \widetilde{\mathcal{L}}_{\bar{E}}(f, f)$, then $(e, s)(f, f)=(e, s)$ gives $e \leq s^{+}$and $s f=s$.

Now suppose that $s g=s$ for some $g \in E$, then

$$
(e, s)(g, g)=\left(e(s g)^{+}, s g\right)=(e, s)
$$

and thus $(f, f)(g, g)=(f, f)$ as $(e, s) \widetilde{\mathcal{L}}_{\bar{E}}(f, f)$. Since $\bar{E} \cong E$, we have $f g=f$, so that $s \widetilde{\mathcal{L}}_{E} f$ using Lemma 1.2 .

Conversely if $e \leq s^{+}$and $s \widetilde{\mathcal{L}}_{E} f$, then

$$
s f=s \Rightarrow(e, s)(f, f)=(e, s)
$$

and if $(e, s)(g, g)=(e, s)$, then $s g=s$ and so $f g=f$, giving

$$
(f, f)(g, g)=(f, f) \text {. }
$$

Hence $(e, s) \widetilde{\mathcal{L}}_{\bar{E}}(f, f)$ using Lemma 1.2 .

(g) It is straightforward to prove that $(g, g) \widetilde{\mathcal{R}}_{\bar{E}}(e, s) \widetilde{\mathcal{L}}_{\bar{E}}(f, f)$ for some $g, f \in E$ implies that $(e, s)=\left(s^{+}, s\right)$ using (c) and (e).

Now let

$$
U=\left\{\left(s^{+}, s\right): s \in S\right\}
$$

To prove that $U$ is a subsemigroup of $Z$, let $\left(s^{+}, s\right),\left(t^{+}, t\right) \in U$, then

$$
\begin{aligned}
\left(s^{+}, s\right)\left(t^{+}, t\right) & =\left(s^{+}\left(s t^{+}\right)^{+}, s t^{+} t\right) \\
& =\left(\left(s^{+} s t\right)^{+}, s t\right) \\
& =\left((s t)^{+}, s t\right) \in U .
\end{aligned}
$$

Clearly $S \cong U$ under $s \mapsto\left(s^{+}, s\right)$, so that $U$ is a left restriction subsemigroup of $Z$ where

$$
\left(s^{+}, s\right)^{+}=\left(\left(s^{+}\right)^{+}, s^{+}\right)=\left(s^{+}, s^{+}\right) \in U \text {. }
$$


We remark that our construction immediately applied to the regular case if $S$ is an inverse semigroup as shown in [6].

The Zappa-Szép product $Z=E \bowtie S$ of a left restriction semigroup $S$ and $E$ is not left restriction in general. The same is true for the semidirect product of a left restriction semigroup and a semilattice. But in the special case where $S$ is a monoid (because monoids are left restriction), it is proved by Fountain et al. [5] that the semidirect product of a monoid with a semilattice is again left restriction. A similar result was proved by Manes [16] in the guise of guarded semigroups and by Gomes and Gould [7] in the case of unipotent monoids for weakly left ample semigroups, where a weakly left ample semigroup is a left restriction semigroup with $E=E(S)$. All of these authors obtained covering theorems of McAlister type for their construction, namely there is an idempotent-separating homomorphism from a subsemigroup of the semidirect product of a monoid with a semilattice to a left restriction semigroup. Although the ZappaSzép product of a semilattice by a left restriction semigroup is not left restriction in general, we nevertheless show that if $S$ is left restriction with semilattice of projections $E$, then we may construct a Zappa-Szép product $Z=E \bowtie S$ in a canonical way as in Proposition 3.3 such that there is an idempotent separating semigroup morphism from $Z$ onto $S$. Indeed, $S$ is a retract of $E \bowtie S$, as is immediate from Proposition 3.3 [(b) and the final statement].

We have shown in Proposition 3.3 that $Z$ contains the left restriction subsemigroup $U$ with semilattice of projections $\bar{E}$, which is isomorphic to $S$. We now find a left restriction subsemigroup $T$ of $Z$ containing $U$, which, in view of Proposition 3.3 (d), is clearly maximum with respect to semilattice of projections $\bar{E}$.

Theorem 3.4 Let $S$ be a left restriction semigroup with semilattice of projections $E$. Suppose that $Z=E \bowtie S$ is the standard Zappa-Szép product of $S$ and E. Let

$$
T=\left\{(e, s): s^{+} \leq e\right\}=\{(e, s): e s=s\}
$$

Then $T$ is the maximum left restriction subsemigroup of $Z$ with semilattice of projections $\bar{E}$.

Let $\beta$ be the restriction to $T$ of the semigroup morphism $\alpha: Z \rightarrow S$ in Proposition 3.3. Then $\beta: T \rightarrow S$ is a projection separating epimorphism of left restriction semigroups.

Proof We first check $T$ is a subsemigroup of $Z=E \bowtie S$. For this let $(e, s),(f, t) \in T$. Then $e s=s, f t=t$, so that

$$
\begin{aligned}
(e, s)(f, t) & =\left(e(s f)^{+}, s f t\right) \\
& =\left((e s f)^{+}, s t\right) \\
& =\left((s f)^{+}, s t\right) .
\end{aligned}
$$

Now $(s f)^{+} s t=s f t=s t$ gives $(e, s)(f, t) \in T$. Hence $T$ is subsemigroup of $Z$. Clearly $\bar{E} \subseteq T$.

To show that $T$ is left restriction, let $(e, s) \in T$. Then $(e, s)^{+} \widetilde{\mathcal{R}}_{\bar{E}}(e, e)$ follows from Proposition 3.3 (c) and the fact that $\bar{E} \subseteq T$. 
Note that for any $(e, s),(f, t) \in T$,

$$
(e, s) \widetilde{\mathcal{R}}_{\bar{E}}(f, t) \Leftrightarrow e=f .
$$

To prove that $\widetilde{\mathcal{R}}_{\bar{E}}$ is a left congruence, let $(e, s),(f, t) \in T$ be such that $(e, s) \widetilde{\mathcal{R}}_{\bar{E}}$ $(f, t)$, that is $e=f$. Then for any $(g, u) \in T$,

$$
((g, u)(e, s))=\left(g(u e)^{+}, u e s\right) \widetilde{\mathcal{R}}_{\bar{E}}\left(g(u f)^{+}, u f t\right)=(g, u)(f, t) .
$$

Therefore $(g, u)(e, s) \widetilde{\mathcal{R}}_{\bar{E}}(g, u)(f, t)$ and so $\widetilde{\mathcal{R}}_{\bar{E}}$ is a left congruence.

Finally to show that the left ample condition holds, let $(e, s) \in T,(f, f) \in \bar{E}$. Then

$$
\begin{array}{rlr}
((e, s)(f, f))^{+}(e, s) & =\left(e(s f)^{+}, s f f\right)^{+}(e, s) \\
& =\left((s f)^{+}, s f\right)^{+}(e, s) \\
& =\left((s f)^{+},(s f)^{+}\right)(e, s) \\
& =\left((s f)^{+}\left((s f)^{+} e\right)^{+},(s f)^{+} e s\right) \\
& =\left((s f)^{+} e,(s f)^{+} s\right) & \\
& =\left(e(s f)^{+}, s f\right) \\
& =(e, s)(f, f) .
\end{array}
$$

Hence $T$ is left restriction with semilattice of projections $\bar{E}$. The fact that $T$ is maximum with respect to these properties comes immediately from Proposition 3.3(d).

Now define $\beta: T \rightarrow S$ by $(e, s) \beta=e s$. Clearly $\beta$ is an epimorphism separating the elements of $\bar{E}$.

Let $\beta: T \rightarrow S$ be as in Theorem 3.4. We note that $\operatorname{Ker} \beta$ intersects trivially with $\widetilde{\mathcal{R}}_{\bar{E}}$. But we cannot expect $T$ to be proper in general as $T$ contains an isomorphic copy of $S$. With a different approach, retaining the same action of $S$ on $E$, but $E$ acting trivially on $S$, we obtain a semidirect product $E \rtimes S$ and this approach yields a proper cover of any left restriction semigroup as proved by Manes [16] (see also $[5,7])$. Manes' left restriction subsemigroup of $E \rtimes S$ is given as follows:

$$
P=\left\{(e, s): e \leq s^{+}\right\}
$$

which becomes a proper cover for $S$ under $(e, s) \mapsto e s$.

If $Z=E \bowtie S$ is the Zappa-Szép product of an inverse semigroup $S$ and its semilattice $E$, then $T=\{(e, s): e s=s\}$ is not inverse. However, from Proposition 3.3 there is a subsemigroup of $T$ which is inverse.

Corollary 3.5 Let $Z=E(S) \bowtie S$ be the standard Zappa-Szép product of an inverse semigroup $S$ and semilattice of idempotents $E(S)$ in the sense of [6] and let $T=$ $\{(e, s):$ es $=s\}$ be a subsemigroup of $Z$. Then there is a canonical embedding of $S$ into $T$ under $s \mapsto\left(s s^{-1}, s\right)$.

We end this paper with couple of examples of Zappa-Szép products. 
The following example is inspired by a result that appeared in [6], where Gilbert and Wazzan considered the Zappa-Szép product $E \bowtie G$ of a group $G$ and a semilattice $E$. By using the natural involution in a group they were able to define a Zappa-Szép product $G \bowtie E$. Left restriction semigroups and monoids do not possess a natural involution in the way that groups and indeed inverse semigroups do. To overcome this, we use the notion of a double action (introduced in [5]) and compatibility conditions (introduced in [3]).

Example 3.6 Let $E \bowtie T$ be a Zappa-Szép product of a semilattice $E$ and a monoid $T$. Define a new action of $T$ on $E$ as follows:

$$
E \times T \rightarrow E,(e, t) \mapsto e \circ t
$$

and suppose that the action of $T$ on $E$ satisfies

$$
e=(t \cdot e) \circ t=t \cdot(e \circ t)
$$

We define a new action of $E$ on $T$ by the following.

$$
e \diamond t=t^{e \circ t}
$$

Then the actions $(e, t) \mapsto e \diamond t$ and $(e, t) \mapsto e \circ t$ satisfy (ZS1)-(ZS4) and we can form a Zappa-Szép product $T \bowtie E$.

Our final example appeared in [10] in context of inverse semigroups.

Example 3.7 Let $X$ be a set and $S=T=\mathcal{P}(X)$ (power set under union). Then $S$ and $T$ are left restriction semigroups where $s^{+}=s$ and $t^{+}=t$, for all $s \in S$ and $t \in T$. Also we note that $S=T$ is a semilattice. For convenience we regard $S$ as a semilattice and $T$ as left restriction semigroup. Define action of $T$ on the left of $S$ by the rule $t \cdot s=t \cup s$ and action of $S$ on the right of $T$ by $t^{s}=t \backslash s$. One can easily verify that (ZS1)-(ZS4) hold and thus $Z=S \bowtie T$ is a Zappa-Szép product.

Acknowledgements The author is grateful to the Schlumberger Foundation for funding her Ph.D. studies, of which this paper forms a part. The author is also grateful to Professor Victoria Gould for her useful suggestions on this manuscript. Also the author would like to thank the referee of this paper whose useful comments helped her in improving the presentation of this article.

Open Access This article is distributed under the terms of the Creative Commons Attribution 4.0 International License (http://creativecommons.org/licenses/by/4.0/), which permits unrestricted use, distribution, and reproduction in any medium, provided you give appropriate credit to the original author(s) and the source, provide a link to the Creative Commons license, and indicate if changes were made.

\section{References}

1. Batbedat, A.: $\gamma$-demi-groupes, demi-modules, produit demi-direct. In: Semi-groups Proceedings: Oberwolfach 1979. Lecture Notes in Mathematics, vol. 855, pp. 1-18. Springer (1981)

2. Batbedat, A., Fountain, J.B.: Connections between left adequate semi-groups and $\gamma$-semigroups. Semigroup Forum 22, 59-65 (1981) 
3. Cornock, C., Gould, V.: Proper restriction semigroups and partial actions. JPAA 216, 935-949 (2012)

4. Cockett, J.R.B., Lack, S.: Restriction categories I: categories of partial maps. Theor. Comput. Sci. 270, 223-259 (2002)

5. Fountain, J.B., Gomes, G.M.S., Gould, V.: The free ample monoid. IJAC 19, 527-554 (2009)

6. Gilbert, N.D., Wazzan, S.: Zappa-Szép products of bands and groups. Semigroup forum 77, 438-455 (2008)

7. Gomes, G.M.S., Gould, V.: Proper weakly left ample semigroups. J. Algebra Comput. 9, 721-739 (1999)

8. Gomes, G.M.S., Gould, V.: Graph expansions of unipotent monoids. Commun. Algebra 28, 447-463 (2000)

9. Gould, V., Hollings, C.: Partial actions of inverse and weakly left E-ample semigroups. J. Aust. Math. Soc. 86, 355-377 (2009)

10. Gould, V., Zenab, R.: Restriction semigroups and $\lambda$-Zappa-Szép products. Period. Math. Hung. 73, 179-207 (2016)

11. Gould, V.: Notes on restriction semigroups and related structures. http://www-users.york.ac.uk/ varg1/ restriction.pdf

12. Howie, J.M.: Fundamentals of Semigroup Theory. Oxford University Press, Oxford (1995)

13. Jackson, M., Stokes, T.: An invitation to C-semigroups. Semigroup Forum 62, 279-310 (2001)

14. Kunze, M.: Zappa products. Acta Math. Hung. 41, 225-239 (1983)

15. Lawson, M.V.: Semigroups and ordered categories. I. The reduced case. J. Algebra 141, 422-462 (1991)

16. Manes, E.: Guarded and banded semigroups. Semigroup Forum 72, 94-120 (2006)

17. McAlister, D.B.: Groups, semilattices and inverse semigroups. Trans. Am. Math. Soc. 192, 227-244 (1974)

18. McAlister, D.B.: Groups, semilattices and inverse semigroups II. Trans. Am. Math. Soc. 196, 351-370 (1974)

19. O’Carroll, L.: Embedding theorems for proper inverse semigroups. J. Algebra 42, 26-40 (1976)

20. Preston, G.B.: Semidirect products of semigroups. Proc. R. Soc. Edinb. 102A, 91-102 (1986)

21. Rédei, L.: Die Anwendung des schiefen Produktes in der Gruppentheorie. J. Reine Angew. Math. 188, 201-227 (1950)

22. Szendrei, M.B.: Proper covers of restriction semigroups and $W$-products. IJAC 22, 16 (2012)

23. Szendrei, M.B.: Embedding into almost left factorizable restriction semigroups. Commun. Algebra 41, 1458-1483 (2013)

24. Trokhimenko, V.S.: Menger's function systems. Izv. Vyš̌. Učebn. Zaved. Mat. 11(138), 71-78 (1973). (Russian)

25. Zappa, G.: Sulla construzione dei gruppi prodotto di due sottogruppi permutabili tra loro. Atti Secondo Congresso Un. Ital. Bologna, pp. 119-125 (1940)

26. Zenab, R.: Decomposition of semigroups into semidirect and Zappa-Szép products, Ph.D. Thesis, University of York (2014) 